\title{
APPROXIMATING SOLUTIONS OF EQUILIBRIUM PROBLEMS IN HADAMARD SPACES
}

\author{
HADI KHATIBZADEH AND VAHID MOHEBBI
}

Received 13 June, 2017

\begin{abstract}
In this paper, we study $\Delta$-convergence and strong convergence of the sequence generated by the extragradient method for pseudo-monotone equilibrium problems in Hadamard spaces. We first show $\Delta$-convergence of the generated sequence to a solution of the equilibrium problem, then the strong convergence of Halpern regularization method is proved. Finally we give some examples where the main results can be applied.
\end{abstract}

2010 Mathematics Subject Classification: 90C33; 74G10

Keywords: Equilibrium problem, extragradient method, Halpern regularization, pseudo-monotone bifunction

\section{Preliminaries}

Let $(X, d)$ be a metric space. For $x, y \in X$, a mapping $c:[0, l] \rightarrow X$, where $l \geq 0$, is called a geodesic with endpoints $x, y$, if $c(0)=x, c(l)=y$, and $d\left(c(t), c\left(t^{\prime}\right)\right)=$ $\left|t-t^{\prime}\right|$ for all $t, t^{\prime} \in[0, l]$. If, for every $x, y \in X$, a geodesic with endpoints $x, y$ exists, then we call $(X, d)$ a geodesic metric space. Furthermore, if there exists a unique geodesic for each $x, y \in X$, then $(X, d)$ is said to be uniquely geodesic.

A subset $K$ of a uniquely geodesic space $X$ is said to be convex when for any two points $x, y \in K$, the geodesic joining $x$ and $y$ is contained in $K$. For each $x, y \in X$, the image of a geodesic $c$ with endpoints $x, y$ is called a geodesic segment joining $x$ and $y$ and is denoted by $[x, y]$.

Let $X$ be a uniquely geodesic metric space. For each $x, y \in X$ and for each $t \in$ $[0,1]$, there exists a unique point $z \in[x, y]$ such that $d(x, z)=t d(x, y)$ and $d(y, z)=$ $(1-t) d(x, y)$. We will use the notation $(1-t) x \oplus t y$ for denoting the unique point $z$ satisfying the above statement.

Definition 1 ([7]). A geodesic space $X$ is called CAT(0) space if for all $x, y, z \in X$ and $t \in[0,1]$ it holds that

$$
d^{2}(t x \oplus(1-t) y, z) \leq t d^{2}(x, z)+(1-t) d^{2}(y, z)-t(1-t) d^{2}(x, y) .
$$

Research for this paper by the second author was supported by CNPq and IMPA. The second author is grateful to CNPq and IMPA for his post-doctoral scholarship. 
A complete $\mathrm{CAT}(0)$ space is called a Hadamard space.

Let $(X, d)$ be a Hadamard space and $\left\{x_{n}\right\}$ be a bounded sequence in $X$. Take $x \in X$. Let $r\left(x,\left\{x_{n}\right\}\right)=\limsup _{n \rightarrow \infty} d\left(x, x_{n}\right)$. The asymptotic radius of $\left\{x_{n}\right\}$ is given by

$$
r\left(\left\{x_{n}\right\}\right)=\inf \left\{r\left(x,\left\{x_{n}\right\}\right) \mid x \in X\right\}
$$

and the asymptotic center of $\left\{x_{n}\right\}$ is the set $A\left(\left\{x_{n}\right\}\right)=\left\{x \in X \mid r\left(x,\left\{x_{n}\right\}\right)=r\left(\left\{x_{n}\right\}\right)\right\}$. It is known that in a Hadamard space, $A\left(\left\{x_{n}\right\}\right)$ consists exactly one point.

Definition 2 (see [23], p. 3690). A sequence $\left\{x_{n}\right\}$ in a Hadamard space $(X, d)$ $\Delta$-converges to $x \in X$ if $A\left(\left\{x_{n_{k}}\right\}\right)=\{x\}$, for each subsequence $\left\{x_{n_{k}}\right\}$ of $\left\{x_{n}\right\}$.

We denote $\Delta$-convergence in $X$ by $\stackrel{\Delta}{\longrightarrow}$ and the metric convergence by $\rightarrow$.

We present next two known results related to the notion of $\Delta$-convergence.

Lemma 1 ([23], Proposition 3.6). Let $X$ be a Hadamard space. Then, every bounded, closed and convex subset of $X$ is $\Delta$-compact; i.e. every bounded sequence in it, has a $\Delta$-convergent subsequence.

Lemma 2 ([7]). Let $(X, d)$ be a $C A T(0)$ space. Then, for all $x, y, z \in X$ and $t \in[0,1]:$

$$
d(t x \oplus(1-t) y, z) \leq t d(x, z)+(1-t) d(y, z) .
$$

Let $C \subset X$ be nonempty, closed and convex. It is well known for any $x \in X$ there exists a unique $u \in C$ such that

$$
d(u, x)=\inf \{d(z, x): z \in C\} .
$$

We define the projection on $C, P_{C}: X \rightarrow C$, by taking as $P_{C}(x)$ the unique $u \in C$ which satisfies (1.1).

A function $h: X \rightarrow(-\infty,+\infty]$ is called:

i) convex iff

$$
h(t x \oplus(1-t) y) \leq t h(x)+(1-t) h(y), \quad \forall x, y \in X \text { and } t \in(0,1)
$$

ii) strictly convex iff

$$
h(t x \oplus(1-t) y)<t h(x)+(1-t) h(y), \quad \forall x, y \in X, x \neq y \text { and } t \in(0,1) .
$$

It is easy to see that each strictly convex function has at most one minimizer on $X$.

Take a closed and convex set $K \subset X$ and $f: X \times X \rightarrow \mathbb{R}$. The equilibrium problem $E P(f, K)$ consists of finding $x^{*} \in K$ such that

$$
f\left(x^{*}, y\right) \geq 0, \quad \forall y \in K .
$$

The set of solutions of $E P(f, K)$ will be denoted as $S(f, K)$.

The equilibrium problem encompasses, among its particular cases, convex optimization problems, variational inequalities (monotone or otherwise), Nash equilibrium problems, and other problems of interest in many applications. 
Equilibrium problems with monotone and pseudo-monotone bifunctions have been studied extensively in Hilbert, Banach as well as in topological vector spaces by many authors (e.g. [3,4,6,12,16]). Recently some authors have studied equilibrium problems on Hadamard manifolds (see [5,28]). The first author and Ranjbar [21] studied a variational inequality for a nonexpansive mapping in Hadamard spaces using the notion of quasi-linearization. This variational inequality is convertable to an equilibrium problem on Hadamard spaces. The authors have studied optimization and equilibrium problems in Hilbert and Hadamard spaces (see [18-20]). In [19] the authors extended some results of pseudo-monotone equilibrium problems and the proximal point algorithm to Hadamard spaces that contain Hilbert spaces and Hadamard manifolds. In this article we study pseudo-monotone equilibrium problems in Hadamard spaces by the extragradient method.

We will deal in this paper with the extragradient (or Korpelevich's) method for equilibrium problems in Hadamard spaces, since it plays an important role in the sequel, we describe it now in some detail. Note that the prototypical example of an equilibrium problem is a variational inequality problem. Therefore we start with an introduction to its well known finite dimensional formulation when applied to variational inequalities, i.e., we assume that $X=\mathbb{R}^{n}$ and $f(x, y)=\langle T(x), y-x\rangle$ with $T: \mathbb{R}^{n} \rightarrow \mathbb{R}^{n}$. Then $\operatorname{EP}(f, K)$ is equivalent to the variational inequality problem $\operatorname{VIP}(T, K)$, consisting of finding a point $x^{*} \in K$ such that $\left\langle T\left(x^{*}\right), y-x^{*}\right\rangle \geq 0$ for all $y \in K$. In this setting, there are several iterative methods for solving $\operatorname{VIP}(T, K)$. In order to solve this problem, Korpelevich suggested in [26] an algorithm of the form:

$$
\begin{gathered}
y_{n}=P_{K}\left(x_{n}-\beta_{n} T\left(x_{n}\right)\right), \\
x_{n+1}=P_{K}\left(x_{n}-\beta_{n} T\left(y_{n}\right)\right),
\end{gathered}
$$

where $P_{K}$ is the projection mapping on $K$ and $\beta_{n}$ is a positive sequence. If $T$ is Lipschitz continuous with constant $L$ and $\operatorname{VIP}(T, K)$ has solutions, then the sequence generated by (1.2)-(1.3) converges to a solution of $V I P(T, K)$ provided that $\beta_{n}=\beta \in(0,1 / L)$ (see [26]). Other variants of Korpelevich's method can be found in $[8,22,27]$.

Extensions of Korpelevich's method to the point-to-set setting (in which case Lipschitz continuity assumption must be carefully reworked, see e.g. [30]), can be found in $[1,13,24,25]$. All these references deal with finite dimensional spaces.

Let $(X, d)$ be a Hadamard space and take $f: X \times X \rightarrow \mathbb{R}$. The following condition on the bifunction $f$ is essential and we will need it in the sequel:

$B_{1}: f(x, \cdot): X \rightarrow \mathbb{R}$ is convex and lower semicontinuous (shortly, lsc) for all $x \in X$.

An extragradient method for equilibrium problems in a Hilbert space $H$ has been studied in [32]. It has the following form: 


$$
\begin{gathered}
y_{n} \in \operatorname{Argmin}_{y \in K}\left\{\beta_{n} f\left(x_{n}, y\right)+\frac{1}{2}\left\|y-x_{n}\right\|^{2}\right\}, \\
x_{n+1} \in \operatorname{Argmin}_{y \in K}\left\{\beta_{n} f\left(y_{n}, y\right)+\frac{1}{2}\left\|y-x_{n}\right\|^{2}\right\},
\end{gathered}
$$

where $\beta_{n}$ is a positive sequence. Weak convergence of the sequence generated by (1.4)-(1.5) to a solution of the equilibrium problem was established in [32]. In [10], this algorithm was upgraded by adding, in each iteration, the orthogonal projection of the initial iterate $x_{0}$ onto the intersection of two halfspaces separating the solution set from the point $x_{n+1}$ given by (1.5). With this upgrade, it was proved in [10] that the sequence of such projections is strongly convergent to a solution of the problem.

It is easy to check that for the variational inequality case, i.e. when $f(x, y)=$ $\langle T(x), y-x\rangle$ for some $T: H \rightarrow H,(1.4)-(1.5)$ reduce precisely to (1.2)-(1.3); indeed the first order optimality condition for the minimization problem in (1.4) (sufficient under convexity of $f\left(x_{n}, \cdot\right)$ and $\left.K\right)$ is $\left\langle\beta_{n} T\left(x_{n}\right)-x_{n}+y, u-y\right\rangle \geq 0$ for all $u \in K$, in which case, by an elementary property of orthogonal projections, $y=P_{K}\left(x_{n}-\right.$ $\left.\beta_{n} T\left(x_{n}\right)\right)$. Since $y_{n}$ satisfies such condition, we have $y_{n}=P_{K}\left(x_{n}-\beta_{n} T\left(x_{n}\right)\right)$, i.e. (1.2). In the same way, (1.5) reduces to (1.3) in the variational inequality case. Thus, it seems reasonable to view (1.4)-(1.5) as an extragradient method for equilibrium problems.

An extragradient method for solving variational inequalities has been studied in [15] and [11]. Then the extragradient method for nonsmooth equilibrium problems in Banach spaces has been recently studied in [14].

In the sequel, let $\varphi: X \rightarrow(-\infty,+\infty]$ be a convex, proper and lsc function where $X$ is a Hadamard space. The resolvent of $\varphi$ of order $\lambda>0$ is defined at each point $x \in X$ as follows

$$
J_{\lambda}^{\varphi} x:=\operatorname{Argmin}_{y \in X}\left\{\varphi(y)+\frac{1}{2 \lambda} d^{2}(y, x)\right\} .
$$

By Lemma 3.1.2 of [17] (see also Lemma 2.2.19 of [2]) for each $x \in X, J_{\lambda}^{\varphi} x$ exists. Therefore we easily conclude the existence of the sequence generated by the extragradient method.

For convergence of the extragradient method, some monotonicity assumptions on the bifunction $f$ are needed. We define next two such properties for future reference: The bifunction $f$ is said to be monotone if $f(x, y)+f(y, x) \leq 0$ for all $x, y \in X$, and pseudo-monotone if for any pair $x, y \in X, f(x, y) \geq 0$ implies $f(y, x) \leq 0$.

We will add now some additional conditions on the bifunction $f$ which will be needed in the convergence analysis:

$B_{2}: f(\cdot, y)$ is $\Delta$-upper semicontinuous for all $y \in X$.

$B_{3}: f$ is Lipschitz-type continuous, i.e. there exist two positive constants $c_{1}$ and $c_{2}$ such that 


$$
f(x, y)+f(y, z) \geq f(x, z)-c_{1} d^{2}(x, y)-c_{2} d^{2}(y, z), \quad \forall x, y, z \in X .
$$

$B_{4}: f$ is pseudo-monotone.

It is well known that a concave and upper semicontinuous function is always $\Delta$ upper semicontinuous.

In this paper we will consider an extragradient method which improves upon (1.4)(1.5) in two senses:

i) We will deal with a rather general class of Hadamard spaces, while [10] only considers Hilbert spaces.

ii) The convergence analysis of the method in [10] requires weak continuity of $f(\cdot, \cdot)$, which seldom holds in infinite dimensional spaces, beyond the case of affine functions. Our continuity assumptions (lower semicontinuity of $f(x, \cdot)$ for all $x \in X$ and $\Delta$-upper semicontinuity of $f(\cdot, y)$ for all $y \in X)$ are much less demanding, and covers the important concave-convex case.

We also mention another difference between our results and those of [10], regarding the additional step required for getting strong convergence, rather than weak. In [10], it consists of a projection of the initial iterate onto the intersection of two halfspaces. Our method, instead, takes a geodesic convex combination in $X$ of the current iterate with a given point in $X$ (see (3.3)).

The paper is organized as follows. In Section 2, we will present an extragradient method for equilibrium problems in Hadamard spaces. We prove $\Delta$-convergence of the generated sequence to a solution of the equilibrium problem, assuming pseudomonotonicity of the bifunction. In Section 3, we propose a variant of the extragradient method for which the generated sequence can be shown to be strongly convergent to an equilibrium point, when the bifunction is pseudo-monotone. In Section 4, we give some examples where the main results can be applied.

\section{EXTRAGRADIENT METHOD AND $\Delta$-CONVERGENCE}

In this section, we study $\Delta$-convergence of the sequence generated by the extragradient method to an equilibrium point of $E P(f, K)$. The method considered in this section is a particular case of the one studied in [32] in finite dimensional Euclidean spaces. We start with a Hadamard space $X$, a closed and convex set $K \subset X$ and a bifunction $f: X \times X \rightarrow \mathbb{R}$. We assume that the bifunction $f$ satisfies $B_{1}, B_{2}, B_{3}$, $B_{4}$ and $S(f, K) \neq \varnothing$, and propose the following Extragradient Method (EM) for solving this problem. Initialize: $x_{0} \in X, n:=0,0<\alpha \leq \lambda_{k} \leq \beta<\min \left\{\frac{1}{2 c_{1}}, \frac{1}{2 c_{2}}\right\}$

and $k=0,1,2, \ldots$

Step 1: Solve the following minimization problem and let $y_{n}$ be the solution of it, i.e.

$$
y_{n} \in \operatorname{Argmin}_{y \in K}\left\{f\left(x_{n}, y\right)+\frac{1}{2 \lambda_{n}} d^{2}\left(x_{n}, y\right)\right\} .
$$


Step 2: Solve the following minimization problem and let $x_{n+1}$ be the solution of it, i.e.

$$
x_{n+1} \in \operatorname{Argmin}_{y \in K}\left\{f\left(y_{n}, y\right)+\frac{1}{2 \lambda_{n}} d^{2}\left(x_{n}, y\right)\right\} .
$$

Step 3: $n:=n+1$ and go back Step 1 .

In order to prove $\Delta$-convergence of the sequences generated by Algorithm EM to an equilibrium point, we need the following lemma.

Lemma 3. Assume that $\left\{x_{n}\right\}$ and $\left\{y_{n}\right\}$ are generated by Algorithm EM and $x^{*} \in$ $S(f, K)$, then

$$
d^{2}\left(x_{n+1}, x^{*}\right) \leq d^{2}\left(x_{n}, x^{*}\right)-\left(1-2 c_{1} \lambda_{n}\right) d^{2}\left(x_{n}, y_{n}\right)-\left(1-2 c_{2} \lambda_{n}\right) d^{2}\left(y_{n}, x_{n+1}\right)
$$

Proof. Take $x^{*} \in S(f, K)$. Note that $x_{n+1}$ solves the minimization problem in (2.2). Now, letting $y=t x_{n+1} \oplus(1-t) x^{*}$ such that $t \in[0,1)$, we have

$$
\begin{aligned}
& f\left(y_{n}, x_{n+1}\right)+\frac{1}{2 \lambda_{n}} d^{2}\left(x_{n}, x_{n+1}\right) \leq f\left(y_{n}, y\right)+\frac{1}{2 \lambda_{n}} d^{2}\left(x_{n}, y\right) \\
& =f\left(y_{n}, t x_{n+1} \oplus(1-t) x^{*}\right)+\frac{1}{2 \lambda_{n}} d^{2}\left(x_{n}, t x_{n+1} \oplus(1-t) x^{*}\right) \\
& \leq t f\left(y_{n}, x_{n+1}\right)+(1-t) f\left(y_{n}, x^{*}\right) \\
& \quad+\frac{1}{2 \lambda_{n}}\left\{t d^{2}\left(x_{n}, x_{n+1}\right)+(1-t) d^{2}\left(x_{n}, x^{*}\right)-t(1-t) d^{2}\left(x_{n+1}, x^{*}\right)\right\} .
\end{aligned}
$$

Since $f\left(x^{*}, y_{n}\right) \geq 0$, pseudo-monotonicity of $f$ implies that $f\left(y_{n}, x^{*}\right) \leq 0$. Hence we can write the above inequality as

$$
f\left(y_{n}, x_{n+1}\right) \leq \frac{1}{2 \lambda_{n}}\left\{d^{2}\left(x_{n}, x^{*}\right)-d^{2}\left(x_{n}, x_{n+1}\right)-t d^{2}\left(x_{n+1}, x^{*}\right)\right\} .
$$

By letting $t \rightarrow 1^{-}$we get

$$
f\left(y_{n}, x_{n+1}\right) \leq \frac{1}{2 \lambda_{n}}\left\{d^{2}\left(x_{n}, x^{*}\right)-d^{2}\left(x_{n}, x_{n+1}\right)-d^{2}\left(x_{n+1}, x^{*}\right)\right\} .
$$

On the other hand, we have

$$
y_{n} \in \operatorname{Argmin}\left\{f\left(x_{n}, y\right)+\frac{1}{2 \lambda_{n}} d^{2}\left(x_{n}, y\right), y \in K\right\} .
$$

Therefore, letting $y=t y_{n} \oplus(1-t) x_{n+1}$ such that $t \in[0,1)$, we obtain

$$
\begin{aligned}
& f\left(x_{n}, y_{n}\right)+\frac{1}{2 \lambda_{n}} d^{2}\left(x_{n}, y_{n}\right) \leq f\left(x_{n}, y\right)+\frac{1}{2 \lambda_{n}} d^{2}\left(x_{n}, y\right) \\
& =f\left(x_{n}, t y_{n} \oplus(1-t) x_{n+1}\right)+\frac{1}{2 \lambda_{n}} d^{2}\left(x_{n}, t y_{n} \oplus(1-t) x_{n+1}\right) \\
& \leq t f\left(x_{n}, y_{n}\right)+(1-t) f\left(x_{n}, x_{n+1}\right)
\end{aligned}
$$




$$
+\frac{1}{2 \lambda_{n}}\left\{t d^{2}\left(x_{n}, y_{n}\right)+(1-t) d^{2}\left(x_{n}, x_{n+1}\right)-t(1-t) d^{2}\left(y_{n}, x_{n+1}\right)\right\} .
$$

Then we receive to

$$
f\left(x_{n}, y_{n}\right)-f\left(x_{n}, x_{n+1}\right) \leq \frac{1}{2 \lambda_{n}}\left\{d^{2}\left(x_{n}, x_{n+1}\right)-d^{2}\left(x_{n}, y_{n}\right)-t d^{2}\left(y_{n}, x_{n+1}\right)\right\} .
$$

Now, if $t \rightarrow 1^{-}$we get

$$
f\left(x_{n}, y_{n}\right)-f\left(x_{n}, x_{n+1}\right) \leq \frac{1}{2 \lambda_{n}}\left\{d^{2}\left(x_{n}, x_{n+1}\right)-d^{2}\left(x_{n}, y_{n}\right)-d^{2}\left(y_{n}, x_{n+1}\right)\right\} .
$$

Also, by $B_{3}, f$ is Lipschitz-type continuous with constants $c_{1}$ and $c_{2}$, hence we have

$$
-c_{1} d^{2}\left(x_{n}, y_{n}\right)-c_{2} d^{2}\left(y_{n}, x_{n+1}\right)+f\left(x_{n}, x_{n+1}\right)-f\left(x_{n}, y_{n}\right) \leq f\left(y_{n}, x_{n+1}\right) \text {. }
$$

Note that by (2.5) and (2.6), we obtain

$$
\left(\frac{1}{2 \lambda_{n}}-c_{1}\right) d^{2}\left(x_{n}, y_{n}\right)+\left(\frac{1}{2 \lambda_{n}}-c_{2}\right) d^{2}\left(y_{n}, x_{n+1}\right)-\frac{1}{2 \lambda_{n}} d^{2}\left(x_{n}, x_{n+1}\right) \leq f\left(y_{n}, x_{n+1}\right) \text {. }
$$

In the sequel by (2.4) and (2.7), we have

$$
\left(1-2 c_{1} \lambda_{n}\right) d^{2}\left(x_{n}, y_{n}\right)+\left(1-2 c_{2} \lambda_{n}\right) d^{2}\left(y_{n}, x_{n+1}\right) \leq d^{2}\left(x_{n}, x^{*}\right)-d^{2}\left(x_{n+1}, x^{*}\right) \text {. }
$$

Remark 1. In Lemma 3, it is obvious that $\lim _{n \rightarrow \infty} d\left(x_{n}, x^{*}\right)$ exists and hence $\left\{x_{n}\right\}$ is bounded. Note that $\liminf _{n \rightarrow \infty}\left(1-2 c_{i} \lambda_{n}\right)>0$ for $i=1,2$. Thus we conclude from Lemma 3 that

$$
\lim _{n \rightarrow \infty} d\left(x_{n}, y_{n}\right)=\lim _{n \rightarrow \infty} d\left(x_{n+1}, y_{n}\right)=\lim _{n \rightarrow \infty} d\left(x_{n}, x_{n+1}\right)=0,
$$

and in the sequel, by (2.4), (2.7) and taking limit, we have $\lim _{n \rightarrow \infty} f\left(y_{n}, x_{n+1}\right)=0$.

Theorem 1. Assume that the bifunction $f$ satisfies $B_{1}, B_{2}, B_{3}$ and $B_{4}$. In addition the solution set $S(f, K)$ is nonempty. Then the sequence $\left\{x_{n}\right\}$ generated by Algorithm $E M, \Delta$-converges to a point of $S(f, K)$.

Proof. Note that $x_{n+1}$ solves the minimization problem in (2.2). By letting $z=$ $t x_{n+1} \oplus(1-t) y$ such that $t \in[0,1)$ and $y \in K$, we get

$$
\begin{aligned}
& f\left(y_{n}, x_{n+1}\right)+\frac{1}{2 \lambda_{n}} d^{2}\left(x_{n}, x_{n+1}\right) \leq f\left(y_{n}, z\right)+\frac{1}{2 \lambda_{n}} d^{2}\left(x_{n}, z\right) \\
& =f\left(y_{n}, t x_{n+1} \oplus(1-t) y\right)+\frac{1}{2 \lambda_{n}} d^{2}\left(x_{n}, t x_{n+1} \oplus(1-t) y\right) \\
& \leq t f\left(y_{n}, x_{n+1}\right)+(1-t) f\left(y_{n}, y\right) \\
& \quad+\frac{1}{2 \lambda_{n}}\left\{t d^{2}\left(x_{n}, x_{n+1}\right)+(1-t) d^{2}\left(x_{n}, y\right)-t(1-t) d^{2}\left(x_{n+1}, y\right)\right\} .
\end{aligned}
$$


Using the above inequality, a straightforward calculation leads to

$$
f\left(y_{n}, x_{n+1}\right)-f\left(y_{n}, y\right) \leq \frac{1}{2 \lambda_{n}}\left\{d^{2}\left(x_{n}, y\right)-d^{2}\left(x_{n}, x_{n+1}\right)-t d^{2}\left(x_{n+1}, y\right)\right\} .
$$

Now, if $t \rightarrow 1^{-}$we obtain

$$
\frac{1}{2 \lambda_{n}}\left\{d^{2}\left(x_{n}, x_{n+1}\right)+d^{2}\left(x_{n+1}, y\right)-d^{2}\left(x_{n}, y\right)\right\} \leq f\left(y_{n}, y\right)-f\left(y_{n}, x_{n+1}\right),
$$

also, it is easy to see that

$$
\frac{-1}{2 \lambda_{n}} d\left(x_{n}, x_{n+1}\right)\left\{d\left(x_{n+1}, y\right)+d\left(x_{n}, y\right)\right\} \leq f\left(y_{n}, y\right)-f\left(y_{n}, x_{n+1}\right) .
$$

Remark 1 shows that $\left\{x_{n}\right\}$ is bounded, therefore there exists a subsequence $\left\{x_{n_{k}}\right\}$ of $\left\{x_{n}\right\}$ and $p \in K$ such that $x_{n_{k}} \stackrel{\Delta}{\longrightarrow} p$ and hence by (2.8), $y_{n_{k}} \stackrel{\Delta}{\longrightarrow} p$. Replacing $n$ by $n_{k}$ in (2.10), taking limsup and using Remark 1 , since $f(\cdot, y)$ is $\Delta$-upper semicontinuous, we have

$$
0 \leq \limsup _{k \rightarrow \infty} f\left(y_{n_{k}}, y\right) \leq f(p, y), \quad \forall y \in K .
$$

Therefore, $p \in S(f, K)$. Finally Opial's Lemma in Hadamard spaces (see Lemma 2.1 in [29]) implies that $\left\{x_{n}\right\} \Delta$-converges to a point of $S(f, K)$.

\section{HALPERN'S REGULARIZATION OF THE EM METHOD}

In this section, we perform a minor modification on the EM algorithm which ensures the strong convergence of the generated sequence to a solution of $E P(f, K)$. In Hilbert spaces, this procedure, called Halpern's regularization (see, e.g., [9]), consists of taking a convex combination of a given EM iterate with a fixed point $u \in X$, where the weight given to $u$ decreases to 0 (see (3.3)). The strong limit of the generated sequence is the projection of $u$ onto the solution set. The modified method will be called HEM. We will assume in the sequel that $X$ is a Hadamard space, $K \subset X$ is closed and convex, and $f: X \times X \rightarrow \mathbb{R}$ is a bifunction which satisfies $B_{1}, B_{2}, B_{3}$, $B_{4}$ and $S(f, K) \neq \varnothing$, and propose the following Halpern's regularization of the Extragradient Method (HEM) for solving this problem.

Initialize: $x_{0}, u \in X, n:=0,0<\alpha \leq \lambda_{k} \leq \beta<\min \left\{\frac{1}{2 c_{1}}, \frac{1}{2 c_{2}}\right\}$ and $k=0,1,2, \ldots$. Take $\left\{\alpha_{k}\right\} \subset(0,1)$ such that $\lim _{k \rightarrow \infty} \alpha_{k}=0$ and $\sum_{k=0}^{\infty} \alpha_{k}=\infty$.

Step 1: Solve the following minimization problem and let $y_{n}$ be the solution of it, i.e.

$$
y_{n} \in \operatorname{Argmin}_{y \in K}\left\{f\left(x_{n}, y\right)+\frac{1}{2 \lambda_{n}} d^{2}\left(x_{n}, y\right)\right\} .
$$


Step 2: Solve the following minimization problem and let $z_{n}$ be the solution of it, i.e.

$$
z_{n} \in \operatorname{Argmin}_{y \in K}\left\{f\left(y_{n}, y\right)+\frac{1}{2 \lambda_{n}} d^{2}\left(x_{n}, y\right)\right\} .
$$

Step 3: Determine the next approximation $x_{n+1}$ as

$$
x_{n+1}=\alpha_{n} u \oplus\left(1-\alpha_{n}\right) z_{n} .
$$

Step 4: $n:=n+1$ and go back Step 1 .

In order to prove the strong convergence result by Algorithm HEM, we need the following lemmas. Although the proof of Lemma 4 is exactly similar to the proof of Lemma 3, but we rewrite it, because we need relations (3.5) and (3.8) to prove our main result in this section.

Lemma 4. Assume that $\left\{x_{n}\right\},\left\{y_{n}\right\}$ and $\left\{z_{n}\right\}$ are generated by Algorithm HEM and $x^{*} \in S(f, K)$, then

$$
d^{2}\left(z_{n}, x^{*}\right) \leq d^{2}\left(x_{n}, x^{*}\right)-\left(1-2 c_{1} \lambda_{n}\right) d^{2}\left(x_{n}, y_{n}\right)-\left(1-2 c_{2} \lambda_{n}\right) d^{2}\left(y_{n}, z_{n}\right)
$$

Proof. Take $x^{*} \in S(f, K)$. Since $z_{n}$ solves the minimization problem in (3.2) by letting $y=t z_{n} \oplus(1-t) x^{*}$ such that $t \in[0,1)$, we have

$$
\begin{aligned}
f\left(y_{n}, z_{n}\right)+\frac{1}{2 \lambda_{n}} d^{2}\left(x_{n}, z_{n}\right) \leq & f\left(y_{n}, y\right)+\frac{1}{2 \lambda_{n}} d^{2}\left(x_{n}, y\right) \\
= & f\left(y_{n}, t z_{n} \oplus(1-t) x^{*}\right)+\frac{1}{2 \lambda_{n}} d^{2}\left(x_{n}, t z_{n} \oplus(1-t) x^{*}\right) \\
\leq & t f\left(y_{n}, z_{n}\right)+(1-t) f\left(y_{n}, x^{*}\right)+\frac{1}{2 \lambda_{n}}\left\{t d^{2}\left(x_{n}, z_{n}\right)\right. \\
& \left.+(1-t) d^{2}\left(x_{n}, x^{*}\right)-t(1-t) d^{2}\left(z_{n}, x^{*}\right)\right\} .
\end{aligned}
$$

Since $f\left(x^{*}, y_{n}\right) \geq 0$, pseudo-monotonicity of $f$ implies that $f\left(y_{n}, x^{*}\right) \leq 0$. Hence we can write the above inequality as

$$
f\left(y_{n}, z_{n}\right) \leq \frac{1}{2 \lambda_{n}}\left\{d^{2}\left(x_{n}, x^{*}\right)-d^{2}\left(x_{n}, z_{n}\right)-t d^{2}\left(z_{n}, x^{*}\right)\right\} .
$$

Now, if $t \rightarrow 1^{-}$, we get

$$
f\left(y_{n}, z_{n}\right) \leq \frac{1}{2 \lambda_{n}}\left\{d^{2}\left(x_{n}, x^{*}\right)-d^{2}\left(x_{n}, z_{n}\right)-d^{2}\left(z_{n}, x^{*}\right)\right\} .
$$

On the other hand, since $y_{n}$ solves the minimization problem in (3.1), by letting $y=t y_{n} \oplus(1-t) z_{n}$ such that $t \in[0,1)$, we have

$$
\begin{aligned}
f\left(x_{n}, y_{n}\right)+\frac{1}{2 \lambda_{n}} d^{2}\left(x_{n}, y_{n}\right) & \leq f\left(x_{n}, y\right)+\frac{1}{2 \lambda_{n}} d^{2}\left(x_{n}, y\right) \\
& =f\left(x_{n}, t y_{n} \oplus(1-t) z_{n}\right)+\frac{1}{2 \lambda_{n}} d^{2}\left(x_{n}, t y_{n} \oplus(1-t) z_{n}\right)
\end{aligned}
$$




$$
\begin{aligned}
\leq & t f\left(x_{n}, y_{n}\right)+(1-t) f\left(x_{n}, z_{n}\right)+\frac{1}{2 \lambda_{n}}\left\{t d^{2}\left(x_{n}, y_{n}\right)\right. \\
& \left.+(1-t) d^{2}\left(x_{n}, z_{n}\right)-t(1-t) d^{2}\left(y_{n}, z_{n}\right)\right\},
\end{aligned}
$$

which implies that

$$
f\left(x_{n}, y_{n}\right)-f\left(x_{n}, z_{n}\right) \leq \frac{1}{2 \lambda_{n}}\left\{d^{2}\left(x_{n}, z_{n}\right)-d^{2}\left(x_{n}, y_{n}\right)-t d^{2}\left(y_{n}, z_{n}\right)\right\} .
$$

Now, if $t \rightarrow 1^{-}$, we get

$$
f\left(x_{n}, y_{n}\right)-f\left(x_{n}, z_{n}\right) \leq \frac{1}{2 \lambda_{n}}\left\{d^{2}\left(x_{n}, z_{n}\right)-d^{2}\left(x_{n}, y_{n}\right)-d^{2}\left(y_{n}, z_{n}\right)\right\} .
$$

Also, by $B_{3}, f$ is Lipschitz-type continuous with constants $c_{1}$ and $c_{2}$, hence we have

$$
-c_{1} d^{2}\left(x_{n}, y_{n}\right)-c_{2} d^{2}\left(y_{n}, z_{n}\right)+f\left(x_{n}, z_{n}\right)-f\left(x_{n}, y_{n}\right) \leq f\left(y_{n}, z_{n}\right) .
$$

Note that by (3.6) and (3.7), we obtain

$$
\left(\frac{1}{2 \lambda_{n}}-c_{1}\right) d^{2}\left(x_{n}, y_{n}\right)+\left(\frac{1}{2 \lambda_{n}}-c_{2}\right) d^{2}\left(y_{n}, z_{n}\right)-\frac{1}{2 \lambda_{n}} d^{2}\left(x_{n}, z_{n}\right) \leq f\left(y_{n}, z_{n}\right) .
$$

In the sequel from (3.5) and (3.8), we conclude that

$$
\left(1-2 c_{1} \lambda_{n}\right) d^{2}\left(x_{n}, y_{n}\right)+\left(1-2 c_{2} \lambda_{n}\right) d^{2}\left(y_{n}, z_{n}\right) \leq d^{2}\left(x_{n}, x^{*}\right)-d^{2}\left(z_{n}, x^{*}\right) .
$$

Lemma 5 ([31]). Let $\left\{s_{n}\right\}$ be a sequence of nonnegative real numbers, $\left\{\alpha_{n}\right\}$ be a sequence of real numbers in $(0,1)$ with $\sum_{n=0}^{\infty} \alpha_{n}=\infty$ and $\left\{t_{n}\right\}$ be a sequence of real numbers. Suppose that

$$
s_{n+1} \leq\left(1-\alpha_{n}\right) s_{n}+\alpha_{n} t_{n}, \quad \forall n \geq 0 .
$$

If limsup $\sup _{k \rightarrow \infty} t_{n_{k}} \leq 0$ for every subsequence $\left\{s_{n_{k}}\right\}$ of $\left\{s_{n}\right\}$ satisfying

$$
\liminf _{k \rightarrow \infty}\left(s_{n_{k}+1}-s_{n_{k}}\right) \geq 0
$$

then $\lim _{n \rightarrow \infty} s_{n}=0$.

Theorem 2. Assume that the bifunction $f$ satisfies $B_{1}, B_{2}, B_{3}$ and $B_{4}$. In addition the solution set $S(f, K)$ is nonempty. Then the sequence $\left\{x_{n}\right\}$ generated by Algorithm HEM converges strongly to $P_{S(f, K)} u$.

Proof. Let $x^{*}=P_{S(f, K)} u$. Lemma 4 shows that

$$
d\left(z_{n}, x^{*}\right) \leq d\left(x_{n}, x^{*}\right) .
$$

By (3.3) and (3.9), we obtain

$$
\begin{aligned}
d\left(x_{n+1}, x^{*}\right) & \leq \alpha_{n} d\left(u, x^{*}\right)+\left(1-\alpha_{n}\right) d\left(z_{n}, x^{*}\right) \\
& \leq \alpha_{n} d\left(u, x^{*}\right)+\left(1-\alpha_{n}\right) d\left(x_{n}, x^{*}\right)
\end{aligned}
$$




$$
\begin{aligned}
& \leq \max \left\{d\left(u, x^{*}\right), d\left(x_{n}, x^{*}\right)\right\} \\
& \leq \cdots \leq \max \left\{d\left(u, x^{*}\right), d\left(x_{0}, x^{*}\right)\right\},
\end{aligned}
$$

which implies that $\left\{x_{n}\right\}$ is bounded. Thus, by (3.9), $\left\{z_{n}\right\}$ is also bounded. On the other hand, (3.3) and (3.9) imply

$$
\begin{aligned}
d^{2}\left(x_{n+1}, x^{*}\right) & \leq\left(1-\alpha_{n}\right) d^{2}\left(z_{n}, x^{*}\right)+\alpha_{n} d^{2}\left(u, x^{*}\right)-\alpha_{n}\left(1-\alpha_{n}\right) d^{2}\left(u, z_{n}\right) \\
& \leq\left(1-\alpha_{n}\right) d^{2}\left(x_{n}, x^{*}\right)+\alpha_{n} d^{2}\left(u, x^{*}\right)-\alpha_{n}\left(1-\alpha_{n}\right) d^{2}\left(u, z_{n}\right) .
\end{aligned}
$$

We are going to prove $d^{2}\left(x_{n}, x^{*}\right) \rightarrow 0$. By Lemma 5 , it suffices to show that

$$
\limsup _{k \rightarrow \infty}\left(d^{2}\left(u, x^{*}\right)-\left(1-\alpha_{n_{k}}\right) d^{2}\left(u, z_{n_{k}}\right)\right) \leq 0
$$

for every subsequence $\left\{d^{2}\left(x_{n_{k}}, x^{*}\right)\right\}$ of $\left\{d^{2}\left(x_{n}, x^{*}\right)\right\}$ satisfying

$$
\liminf _{k \rightarrow \infty}\left(d^{2}\left(x_{n_{k}+1}, x^{*}\right)-d^{2}\left(x_{n_{k}}, x^{*}\right)\right) \geq 0
$$

Consider such a sequence. We have

$$
\begin{aligned}
0 & \leq \liminf _{k \rightarrow \infty}\left(d^{2}\left(x_{n_{k}+1}, x^{*}\right)-d^{2}\left(x_{n_{k}}, x^{*}\right)\right) \\
& \leq \liminf _{k \rightarrow \infty}\left(\alpha_{n_{k}} d^{2}\left(u, x^{*}\right)+\left(1-\alpha_{n_{k}}\right) d^{2}\left(z_{n_{k}}, x^{*}\right)-d^{2}\left(x_{n_{k}}, x^{*}\right)\right) \\
& =\liminf _{k \rightarrow \infty}\left(\alpha_{n_{k}}\left(d^{2}\left(u, x^{*}\right)-d^{2}\left(z_{n_{k}}, x^{*}\right)\right)+d^{2}\left(z_{n_{k}}, x^{*}\right)-d^{2}\left(x_{n_{k}}, x^{*}\right)\right) \\
& \leq \limsup _{k \rightarrow \infty} \alpha_{n_{k}}\left(d^{2}\left(u, x^{*}\right)-d^{2}\left(z_{n_{k}}, x^{*}\right)\right)+\liminf _{k \rightarrow \infty}\left(d^{2}\left(z_{n_{k}}, x^{*}\right)-d^{2}\left(x_{n_{k}}, x^{*}\right)\right) \\
& =\liminf _{k \rightarrow \infty}\left(d^{2}\left(z_{n_{k}}, x^{*}\right)-d^{2}\left(x_{n_{k}}, x^{*}\right)\right) \\
& \leq \limsup _{k \rightarrow \infty}\left(d^{2}\left(z_{n_{k}}, x^{*}\right)-d^{2}\left(x_{n_{k}}, x^{*}\right)\right) \leq 0 .
\end{aligned}
$$

This shows that $\lim _{k \rightarrow \infty}\left(d^{2}\left(z_{n_{k}}, x^{*}\right)-d^{2}\left(x_{n_{k}}, x^{*}\right)\right)=0$, i.e.

$$
\lim _{k \rightarrow \infty} d^{2}\left(x_{n_{k}}, x^{*}\right)=\lim _{k \rightarrow \infty} d^{2}\left(z_{n_{k}}, x^{*}\right)
$$

Replacing $n$ by $n_{k}$ in Lemma 4, since $\liminf _{n \rightarrow \infty}\left(1-2 c_{i} \lambda_{n}\right)>0$ for $i=1$, 2, we conclude that

$$
\lim _{k \rightarrow \infty} d^{2}\left(x_{n_{k}}, y_{n_{k}}\right)=\lim _{k \rightarrow \infty} d^{2}\left(y_{n_{k}}, z_{n_{k}}\right)=\lim _{k \rightarrow \infty} d^{2}\left(x_{n_{k}}, z_{n_{k}}\right)=0 .
$$

Now, replacing $n$ by $n_{k}$ in (3.5) and (3.8), and taking limit, we get

$$
\lim _{k \rightarrow \infty} f\left(y_{n_{k}}, z_{n_{k}}\right)=0 .
$$


On the other hand, there exists a subsequence $\left\{z_{n_{k_{t}}}\right\}$ of $\left\{z_{n_{k}}\right\}$ and $p \in K$ such that $z_{n_{k_{t}}} \stackrel{\Delta}{\longrightarrow} p$ and

$\limsup _{k \rightarrow \infty}\left(d^{2}\left(u, x^{*}\right)-\left(1-\alpha_{n_{k}}\right) d^{2}\left(u, z_{n_{k}}\right)\right)=\lim _{t \rightarrow \infty}\left(d^{2}\left(u, x^{*}\right)-\left(1-\alpha_{n_{k_{t}}}\right) d^{2}\left(u, z_{n_{k_{t}}}\right)\right)$.

By $\Delta$-lower semicontinuity of $d^{2}(u, \cdot)$, we obtain

$$
\begin{aligned}
& \limsup _{k \rightarrow \infty}\left(d^{2}\left(u, x^{*}\right)-\left(1-\alpha_{n_{k}}\right) d^{2}\left(u, z_{n_{k}}\right)\right) \\
= & \lim _{t \rightarrow \infty}\left(d^{2}\left(u, x^{*}\right)-\left(1-\alpha_{n_{k_{t}}}\right) d^{2}\left(u, z_{n_{k_{t}}}\right)\right) \\
\leq & d^{2}\left(u, x^{*}\right)-d^{2}(u, p) .
\end{aligned}
$$

Now, note that since $z_{n}$ solves the minimization problem in (3.2) by letting $z=$ $t z_{n} \oplus(1-t) y$ such that $t \in[0,1)$ and $y \in K$, we have

$$
\begin{aligned}
f\left(y_{n}, z_{n}\right)+\frac{1}{2 \lambda_{n}} d^{2}\left(x_{n}, z_{n}\right) \leq & f\left(y_{n}, z\right)+\frac{1}{2 \lambda_{n}} d^{2}\left(x_{n}, z\right) \\
= & f\left(y_{n}, t z_{n} \oplus(1-t) y\right)+\frac{1}{2 \lambda_{n}} d^{2}\left(x_{n}, t z_{n} \oplus(1-t) y\right) \\
\leq & t f\left(y_{n}, z_{n}\right)+(1-t) f\left(y_{n}, y\right)+\frac{1}{2 \lambda_{n}}\left\{t d^{2}\left(x_{n}, z_{n}\right)\right. \\
& \left.+(1-t) d^{2}\left(x_{n}, y\right)-t(1-t) d^{2}\left(z_{n}, y\right)\right\} .
\end{aligned}
$$

A straightforward calculation leads to

$$
f\left(y_{n}, z_{n}\right)-f\left(y_{n}, y\right) \leq \frac{1}{2 \lambda_{n}}\left\{d^{2}\left(x_{n}, y\right)-d^{2}\left(x_{n}, z_{n}\right)-t d^{2}\left(z_{n}, y\right)\right\} .
$$

Now, if $t \rightarrow 1^{-}$we obtain

$$
\frac{1}{2 \lambda_{n}}\left\{d^{2}\left(x_{n}, z_{n}\right)+d^{2}\left(z_{n}, y\right)-d^{2}\left(x_{n}, y\right)\right\} \leq f\left(y_{n}, y\right)-f\left(y_{n}, z_{n}\right) .
$$

It is easy to see that

$$
\frac{-1}{2 \lambda_{n}} d\left(x_{n}, z_{n}\right)\left\{d\left(z_{n}, y\right)+d\left(x_{n}, y\right)\right\} \leq f\left(y_{n}, y\right)-f\left(y_{n}, z_{n}\right) .
$$

Since $y_{n_{k_{t}}} \stackrel{\Delta}{\longrightarrow} p$, replacing $n$ by $n_{k_{t}}$ in (3.16) and then taking limsup and using (3.12) and (3.13) we get

$$
0 \leq \limsup _{t \rightarrow \infty} f\left(y_{n_{k_{t}}}, y\right), \quad \forall y \in K .
$$

Now, since $f(\cdot, y)$ is $\Delta$-upper semicontinuous, we get

$$
f(p, y) \geq 0, \quad \forall y \in K
$$


i.e. $p \in S(f, K)$. Therefore we have $d\left(u, x^{*}\right) \leq d(u, p)$ by the definition of $x^{*}$, thus (3.14) implies that

$$
\limsup _{k \rightarrow \infty}\left(d^{2}\left(u, x^{*}\right)-\left(1-\alpha_{n_{k}}\right) d^{2}\left(u, z_{n_{k}}\right)\right) \leq 0 .
$$

Hence

$$
d^{2}\left(x_{n}, x^{*}\right) \rightarrow 0
$$

i.e. $x_{n} \rightarrow x^{*}=P_{S(f, K)} u$.

\section{SOME EXAMPLES AND APPLICATIONS}

In this section, in order to show some applications of our main results, we give some examples of equilibrium problems in Hadamard spaces. Hilbert spaces and $\mathbb{R}$ trees are two basic examples of Hadamard spaces, which in some sense represent the most extreme cases; curvature 0 and curvature $-\infty$. The most illuminating instances of Hadamard spaces are Hadamard manifolds. A Hadamard manifold is a complete simply connected Riemannian manifold of nonpositive sectional curvature. The class of Hadamard manifolds includes hyperbolic spaces, manifolds of positive definite matrices, the complex Hilbert ball with the hyperbolic metric and etc. The details are described in [2].

In the sequel, we first recall hyperbolic spaces. We equip $\mathbb{R}^{n+1}$ with the inner product

$$
\langle x, y\rangle=-x_{0} y_{0}+\sum_{i=1}^{n} x_{i} y_{i},
$$

for $x=\left(x_{0}, x_{1}, \ldots, x_{n}\right)$ and $y=\left(y_{0}, y_{1}, \ldots, y_{n}\right)$. Define

$$
\mathbb{T}^{n}:=\left\{x=\left(x_{0}, x_{1}, \ldots, x_{n}\right) \in \mathbb{R}^{n+1}:\langle x, x\rangle=-1, x_{0}>0\right\} .
$$

Then $\langle\cdot, \cdot\rangle$ induces the Riemannian metric $d$ on the tangent spaces $T_{p} \mathbb{U}^{n} \subset T_{p} \mathbb{R}^{n+1}$ as

$$
d(x, y)=\operatorname{arccosh}(-\langle x, y\rangle), \quad \forall x, y \in \mathbb{H}^{n}
$$

for $p \in \mathbb{Q}^{n}$. Then $\left(\mathbb{\mathbb { H } ^ { n }}, d\right)$ is Hadamard manifold with sectional curvature -1 at every point (see [2]).

In the following example, we give an equilibrium problem in the hyperbolic space.

Example 1. Let $X=\mathbb{G}^{n}$ be the hyperbolic space and $T: X \rightarrow X$ is the nonexpansive mapping defined by

$$
T(x)=\left(x_{0},-x_{1},-x_{2}, \ldots,-x_{n}\right) .
$$

Now, we define $f: X \times X \rightarrow \mathbb{R}$ as $f(x, y)=\langle x-T(x), y-x\rangle$. If $x$ is an equilibrium point of $E P(f, X)$, then $f(x, y) \geq 0$ for all $y \in X$. Taking $y=T(x)$, we get

$$
\langle x-T(x), T(x)-x\rangle \geq 0,
$$


which implies that $-4 x_{1}^{2}-4 x_{2}^{2}-\cdots-4 x_{n}^{2} \geq 0$, i.e. $x_{1}=x_{2}=\cdots=x_{n}=0$. On the other hand, we have $\langle x, x\rangle=-1$. Therefore we conclude that $x_{0}=1$. Hence $(1,0,0, \ldots, 0)$ is an equilibrium point of $E P(f, X)$ and it is also a fixed point for $T$.

The space $P(n, \mathbb{R})$ of symmetric positive definite matrices $n \times n$ with real entries is a Hadamard manifold if it is equipped with the Riemannian metric

$$
\langle X, Y\rangle_{A}:=\operatorname{Tr}\left(A^{-1} X A^{-1} Y\right), \quad \forall X, Y \in T_{A}(P(n, \mathbb{R}))
$$

for every $A \in P(n, \mathbb{R})$.

Example 2. Consider $P(n, \mathbb{R})$ with $n=1$, i.e. the positive real numbers $\mathbb{R}^{+}$along with the inner product $\langle x, y\rangle_{a}=\frac{x y}{a^{2}}$ for $a \in \mathbb{R}^{+}$and $x, y \in T_{a} \mathbb{R}^{+}=\mathbb{R}$. The metric on $\mathbb{R}^{+}$is defined as $d(a, b)=|\ln a-\ln b|$ and $\gamma(t)=a\left(\frac{b}{a}\right)^{t}$ is the geodesic between $a, b \in \mathbb{R}^{+}$. Therefore $X=\mathbb{R}^{+}$with this metric is a CAT(0) space and the inequality in Definition 1 becomes equality. If $\gamma(t)=a\left(\frac{b}{a}\right)^{t}$ is the geodesic between $a, b \in \mathbb{R}^{+}$, we have

$$
\ln \gamma(t)=\ln a\left(\frac{b}{a}\right)^{t}=\ln a+t(\ln b-\ln a)=(1-t) \ln a+t \ln b .
$$

Now, we define $f: X \times X \rightarrow \mathbb{R}$ as $f(x, y)=(\ln x)(\ln y)-(\ln x)^{2}$. Note that

$$
f(x, \gamma(t))=(\ln x)(\ln \gamma(t))-(\ln x)^{2}=(1-t) f(x, a)+t f(x, b) .
$$

It is obvious that $f$ satisfies $B_{2}$. Now we show that $f$ satisfies $B_{3}$ with Lipschitz constants $c_{1}=c_{2}=\frac{1}{2}$. Take $x, y, z \in X$, then we have

$$
\begin{aligned}
f(x, y)+f(y, z)-f(x, z) & =(\ln x)(\ln y)+(\ln y)(\ln z)-(\ln y)^{2}-(\ln x)(\ln z) \\
& =(\ln x)(\ln y-\ln z)-(\ln y)(\ln y-\ln z) \\
& =(\ln x-\ln y)(\ln y-\ln z) \\
& \geq-d(x, y) d(y, z) \\
& \geq-\frac{1}{2} d^{2}(x, y)-\frac{1}{2} d^{2}(y, z) .
\end{aligned}
$$

Also, the following statement shows that $f$ is monotone

$$
\begin{aligned}
f(x, y)+f(y, x) & =(\ln x)(\ln y)-(\ln x)^{2}+(\ln y)(\ln x)-(\ln y)^{2} \\
& =-(\ln x-\ln y)^{2} \leq 0 .
\end{aligned}
$$

Finally, if we consider $K=[2,+\infty)$, then $S(f, K) \neq \varnothing$.

In the following example, we give a class of pseudo-monotone bifunctions.

Example 3. Suppose that $(X, d)$ is a Hadamard space. Let $\varphi: X \rightarrow \mathbb{R}^{+}$and $\psi$ : $X \rightarrow \mathbb{R}$ be two functions. We define $f: X \times X \rightarrow \mathbb{R}$ as $f(x, y)=\varphi(x)(\psi(y)-$ 
$\psi(x))$. It is clear that $f$ is pseudo-monotone bifunction. Now, if there exist $c_{1}>0$ and $c_{2}>0$ such that

$$
(\varphi(x)-\varphi(y))(\psi(y)-\psi(z)) \geq-c_{1} d^{2}(x, y)-c_{2} d^{2}(y, z),
$$

then $f$ satisfies $B_{3}$.

In the last example, we first introduce the geometric median and the Fréchet mean for a finite set of points in Hadamard spaces. Then we define two bifunctions $f_{j}$ for $j=1,2$, which satisfy $B_{1}, B_{2}, B_{3}, B_{4}$ and their equilibrium points are the geometric median or the Fréchet mean of the finite set of points. This result can be used for finding median and mean in a Hadamard space.

Example 4. Let $w_{1}, \ldots, w_{n}$ be positive weights satisfying $\sum_{i=1}^{n} w_{i}=1$ and $z_{1}, \ldots, z_{n}$ be in a Hadamard space $(X, d)$. We define the geometric median for $z_{1}, \ldots, z_{n}$ as

$$
\operatorname{Argmin}\left\{\sum_{i=1}^{n} w_{i} d\left(x, z_{i}\right): x \in X\right\}
$$

and the Fréchet mean as

$$
\operatorname{Argmin}\left\{\sum_{i=1}^{n} w_{i} d^{2}\left(x, z_{i}\right): x \in X\right\} .
$$

Now, we define $\phi_{j}: X \rightarrow \mathbb{R}$ as $\phi_{j}(x)=\sum_{i=1}^{n} w_{i} d^{j}\left(x, z_{i}\right)$ and then we consider $f_{j}: X \times X \rightarrow \mathbb{R}$ as $f_{j}(x, y)=\phi_{j}(y)-\phi_{j}(x)$ for $j=1,2$. It is obvious that $f_{j}$ satisfies $B_{1}, B_{2}, B_{3}, B_{4}$ and $S\left(f_{j}, X\right) \neq \varnothing$, and any equilibrium point of $E P\left(f_{j}, X\right)$ is the minimum point of $\phi_{j}$ for $j=1,2$.

\section{ACKNOWLEDGEMENT}

The authors are grateful to the referee for his/her valuable comments and suggestions.

\section{REFERENCES}

[1] T. Q. Bao and P. Q. Khanh, A projection-type algorithm for pseudomonotone nonlipschitzian multivalued variational inequalities. New York, NY: Springer, 2005. doi: 10.1007/0-387-23639-2-6.

[2] M. Bačák, Convex analysis and optimization in Hadamard spaces. Berlin: De Gruyter, 2014, vol. 22, doi: 10.1515/9783110361629.

[3] M. Bianchi and S. Schaible, "Generalized monotone bifunctions and equilibrium problems." $J$. Optim. Theory Appl., vol. 90, no. 1, pp. 31-43, 1996, doi: 10.1007/BF02192244.

[4] O. Chadli, Z. Chbani, and H. Riahi, "Equilibrium problems with generalized monotone bifunctions and applications to variational inequalities." J. Optim. Theory Appl., vol. 105, no. 2, pp. 299-323, 2000.

[5] V. Colao, G. López, G. Marino, and V. Martín-Márquez, "Equilibrium problems in Hadamard manifolds," J. Math. Anal. Appl., vol. 388, no. 1, pp. 61-77, 2012, doi: 10.1016/j.jmaa.2011.11.001. 
[6] P. L. Combettes and S. A. Hirstoaga, "Equilibrium programming in Hilbert spaces," J. Nonlinear Convex Anal., vol. 6, no. 1, pp. 117-136, 2005.

[7] S. Dhompongsa and B. Panyanak, "On $\Delta$-convergence theorems in CAT(0) spaces," Comput. Math. Appl., vol. 56, no. 10, pp. 2572-2579, 2008, doi: 10.1016/j.camwa.2008.05.036.

[8] E. G. Golshtein and N. V. Tretyakov, Modified Lagrangians and monotone maps in optimization. Transl. from the Russian by N. V. Tretyakov. New York, NY: Wiley, 1996.

[9] B. Halpern, "Fixed points of nonexpanding maps." Bull. Am. Math. Soc., vol. 73, pp. 957-961, 1967, doi: 10.1090/S0002-9904-1967-11864-0.

[10] D. V. Hieu, "Common solutions to pseudomonotone equilibrium problems." Bull. Iran. Math. Soc., vol. 42, no. 5, pp. 1207-1219, 2016.

[11] A. N. Iusem and B. F. Svaiter, "A variant of Korpelevich's method for variational inequalities with a new search strategy." Optimization, vol. 42, no. 4, pp. 309-321, 1997, doi: 10.1080/02331939708844365.

[12] A. N. Iusem, G. Kassay, and W. Sosa, "On certain conditions for the existence of solutions of equilibrium problems.” Math. Program., vol. 116, no. 1-2 (B), pp. 259-273, 2009, doi: 10.1007/s10107-007-0125-5.

[13] A. N. Iusem and L. R. Lucambio Pérez, "An extragradient-type algorithm for nonsmooth variational inequalities.” Optimization, vol. 48, no. 3, pp. 309-332, 2000, doi: 10.1080/02331930008844508.

[14] A. N. Iusem and V. Mohebbi, "Extragradient method for nonsmooth equilibrium problems in Banach spaces," Optimization, 2018, doi: 10.1080/02331934.2018.1462808.

[15] A. N. Iusem and M. Nasri, "Korpelevich's method for variational inequality problems in Banach spaces," J. Global Optim., vol. 50, no. 1, pp. 59-76, 2011, doi: 10.1007/s10898-010-9613-x.

[16] A. N. Iusem and W. Sosa, "On the proximal point method for equilibrium problems in Hilbert spaces." Optimization, vol. 59, no. 7-8, pp. 1259-1274, 2010, doi: 10.1080/02331931003603133.

[17] J. Jost, Nonpositive curvature: geometric and analytic aspects. Basel: Birkhäuser, $1997 . \quad$ doi: 10.1007/978-3-0348-8918-6.

[18] H. Khatibzadeh and V. Mohebbi, "Proximal point algorithm for infinite pseudo-monotone bifunctions." Optimization, vol. 65, no. 8, pp. 1629-1639, 2016, doi: 10.1080/02331934.2016.1153639.

[19] H. Khatibzadeh and V. Mohebbi, "Monotone and pseudo-monotone equilibrium problems in Hadamard spaces." J. Aust. Math. Soc., 2019, doi: 10.1017/S1446788719000041.

[20] H. Khatibzadeh, V. Mohebbi, and S. Ranjbar, "New results on the proximal point algorithm in non-positive curvature metric spaces." Optimization, vol. 66, no. 7, pp. 1191-1199, 2017, doi: 10.1080/02331934.2017.1326044.

[21] H. Khatibzadeh and S. Ranjbar, "A variational inequality in complete CAT(0) spaces." J. Fixed Point Theory Appl., vol. 17, no. 3, pp. 557-574, 2015, doi: 10.1007/s11784-015-0245-0.

[22] E. N. Khobotov, "A modification of the extragradient method for solving variational inequalities and some optimization problems," Zh. Vychisl. Mat. i Mat. Fiz., vol. 27, no. 10, pp. 1462-1473, 1597, 1987.

[23] W. A. Kirk and B. Panyanak, "A concept of convergence in geodesic spaces." Nonlinear Anal., Theory Methods Appl., Ser. A, Theory Methods, vol. 68, no. 12, pp. 3689-3696, 2008, doi: 10.1016/j.na.2007.04.011.

[24] I. V. Konnov, “On combined relaxation methods' convergence rates.” Russ. Math., vol. 37, no. 12, pp. 89-92, 1993.

[25] I. Konnov, Combined relaxation methods for variational inequalities. Berlin: Springer, 2001, vol. 495, doi: 10.1007/978-3-642-56886-2.

[26] G. M. Korpelevich, "An extragradient method for finding saddle points and for other problems." Mat. Metody Resheniya Èkon. Zadach, vol. 12, pp. 747-756, 1976. 
[27] P. Marcotte, "Application of Khobotov's algorithm to variational inequalities and network equilibrium problems." INFOR, vol. 29, no. 4, pp. 258-270, 1991.

[28] M. A. Noor and K. I. Noor, "Some algorithms for equilibrium problems on Hadamard manifolds." J. Inequal. Appl., vol. 2012, p. 8, 2012, doi: 10.1186/1029-242X-2012-230.

[29] S. Ranjbar and H. Khatibzadeh, " $\Delta$-convergence and $w$-convergence of the modified Mann iteration for a family of asymptotically nonexpansive type mappings in complete CAT(0) spaces." Fixed Point Theory, vol. 17, no. 1, pp. 151-158, 2016.

[30] S. M. Robinson and S. Lu, "Solution continuity in variational conditions." J. Glob. Optim., vol. 40, no. 1-3, pp. 405-415, 2008, doi: 10.1007/s10898-007-9192-7.

[31] S. Saejung and P. Yotkaew, "Approximation of zeros of inverse strongly monotone operators in Banach spaces." Nonlinear Anal., Theory Methods Appl., Ser. A, Theory Methods, vol. 75, no. 2, pp. 742-750, 2012, doi: 10.1016/j.na.2011.09.005.

[32] D. Q. Tran, M. L. Dung, and V. H. Nguyen, "Extragradient algorithms extended to equilibrium problems." Optimization, vol. 57, no. 6, pp. 749-776, 2008, doi: 10.1080/02331930601122876.

Authors' addresses

Hadi Khatibzadeh

University of Zanjan, Department of Mathematics, P. O. Box 45195-313, Zanjan, Iran

E-mail address: hkhatibzadeheznu.ac.ir

\section{Vahid Mohebbi}

Instituto de Matemática Pura e Aplicada, Estrada Dona Castorina 110, RJ, 22460-320, Rio de Janeiro, Brazil

Current address: University of Texas at El Paso, Department of Mathematical Sciences, 500 W. University Avenue, 79968 El Paso, Texas, USA

E-mail address: mohebbi@impa.br, vmohebbi@utep.edu 